NETO, E. M. R.; MARQUES, L. A. R. V.; LOTIF, M. A. L.; COELHO, M. O.; NOCRATO, M. N.; RODRIGUES, J. C.

Revista Eletrônica de Farmácia Vol. X (4), 16 - 26, 2013.

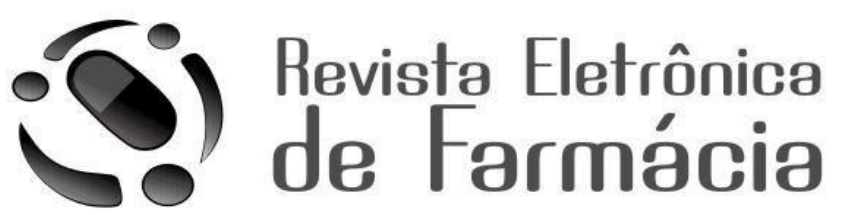

REF - ISSN 1808-0804 Vol. X (4), 16 - 26, 2013.

\title{
DERMATITE SEBORRÉICA: ABORDAGEM TERAPÊUTICA NO ÂMBITO DA CLÍNICA FARMACÊUTICA
}

SEBORRHEIC DERMATITIS: THERAPEUTIC APPROACH IN PHARMACEUTICAL CLINICAL

DERMATITIS SEBORREICA: ABORDAJE TERAPÉUTICA EN LA FARMACIA CLÍNICA

\begin{abstract}
Edilson Martins Rodrigues Neto; Lidia Audrey Rocha Valadas Marques; Mara Assef Leitão Lotif; Marcia Oliveira Coelho; Miriam Nuces Nocrato; Jessica Cavalcante Rodrigues.
\end{abstract}

Recebido em 24/11/2013, Aceito em 28/12/2013.

RESUMO: A dermatite seborréica é uma doença de pele muito comum caracterizada por cronicidade, inflamação e descamação epidérmica. Considerando que é uma condição patológica sem cura definitiva cabe ao farmacêutico esclarecer ao paciente que os objetivos do tratamento são controlar os sinais visíveis das lesões e sintomas incômodos como o prurido. Assim, o presente estudo objetivou realizar uma revisão de literatura visando nortear a abordagem terapêutica da Dermatite Seborréica no âmbito competente da clínica farmacêutica. Foi realizada uma revisão de literatura com pesquisa nos bancos 
NETO, E. M. R.; MARQUES, L. A. R. V.; LOTIF, M. A. L.; COELHO, M. O.; NOCRATO, M. N.; RODRIGUES, J. C.

Revista Eletrônica de Farmácia Vol. X (4), 16 - 26, 2013.

de dados do Pubmed, Bireme, Lilacs e Scielo, bem como pesquisa nos periódicos do Portal Brasileiro da Informação Científica. Foram usados os descritores: Dermatite Seborréica, Tratamento Farmacológico e Malassezia e selecionados artigos relacionados à abordagem terapêutica da dermatite seborreica tendo como foco a competência profissional do farmacêutico. Visto que, muitas vezes, o farmacêutico é o primeiro profissional de saúde que o paciente procura, é de suma importância que, dentro da sua competência clínica, ele seja capaz de resolver um problema de saúde menor ou encaminhar o paciente para atendimento específico. Com a farmacoterapia e mudanças de hábitos é possível se obter um controle efetivo e um maior espaçamento na recorrência das crises da doença.

PALAVRAS-CHAVE: Dermatite Seborreica; Malassezia; Prescrições de Medicamentos.

ABSTRACT: Seborrheic dermatitis is a very common skin disease characterized by chronic inflammation and epidermal desquamation. Whereas it is a pathological condition with no permanent cure it is for the pharmacist to clarify the patient that the goals of treatment are to control the visible signs of injuries and troublesome symptoms such as itching. Thus, the present study aimed to perform a literature review aimed at guiding the therapeutic management of Seborrheic Dermatitis competent in the pharmaceuticals clinic. A literature review was performed with research in databases Pubmed, Bireme, Lilacs and SciELO, as well as research in the journals of the Brazilian Portal of Scientific Information. Were used the descriptors Seborrheic Dermatitis, Pharmacological Treatment and Malassezia and selected scientific articles related to the therapeutic approach of seborrheic dermatitis focusing on the professional competence of pharmacist. As often the pharmacist is the first health care professional that the patient demand, is very important, within their clinical competence, he is able to solve a minor health issue or refer the patient for specific care. With pharmacotherapy and changing habits is possible to obtain effective control and greater spacing in the recurrence of seizures of this disease.

KEYWORDS: Seborrheic Dermatitis; Malassezia; Drug Prescriptions.

RESUMEN: La dermatitis seborreica es una enfermedad muy común de la piel caracterizada por cronicidad, inflamación y descamación epidérmica. Teniendo en cuenta que es una condición patológica sin una cura definitiva, es una función del farmacéutico aclarar al paciente que los objetivos del tratamiento son controlar los signos visibles de 
NETO, E. M. R.; MARQUES, L. A. R. V.; LOTIF, M. A. L.; COELHO, M. O.; NOCRATO, M. N.; RODRIGUES, J. C.

Revista Eletrônica de Farmácia Vol. X (4), 16 - 26, 2013.

lesiones y síntomas incómodos como picazón. Así, el presente estudio pretende llevar a cabo una revisión de literatura a fin de orientar el enfoque terapéutico de la Dermatitis Seborreica en el contexto de clínica farmacéutica. Se realizó una revisión de la literatura con la búsqueda en las bases de datos Pubmed, Bireme, LILACS y SciELO, así como investigación en publicaciones periódicas del Portal Brasileño de Información Científica. Se utilizaron los descriptores: Dermatitis Seborreica, Tratamiento Farmacológico y Malassezia y fueron seleccionados artículos con el enfoque terapéutico de la dermatitis seborreica, centrándose en la competencia profesional del farmacéutico. Visto que, a menudo, el farmacéutico es el primero profesional de la salud que el paciente busca, es de suma importancia que, dentro de sus competéncias clínicas, sea capaz resolver un problema de salud menor o referir al paciente a un servicio específico. Con la farmacoterapia y los cambios en los hábitos es posible obtener un control eficaz y un mayor espaciamento en la recurrencia de crisis de la enfermedad.

PALABRAS-CLAVE: Dermatitis Seborreica; Malassezia; Prescripciones de Medicamentos.

\section{INTRODUÇÃO}

A Dermatite Seborréica (DS) é uma doença de pele de caráter inflamatório e crônico que se manifesta inicialmente por lesões eritematosas, bem delimitadas, cobertas por escamas gordurosas ou placas róseas à amareladas de formatos e tamanhos variados; essas lesões eritemodescamativas desenvolvem-se, principalmente, em regiões ricas em glândulas sebáceas, como por exemplo o couro cabeludo, face, tórax e flexuras. ${ }^{(1)}$

Essa doença atinge 2 a $5 \%$ da população mundial e afeta uma ampla faixa etária, desde recém-nascidos ate a fase senil, porém possui maior densidade de incidência em adultos e idosos. ${ }^{(1)}$
Dados epidemiológicos dos Estados Unidos da America revelam que cerca de 3 a $5 \%$ da população é acometida, sendo maior sua incidência em pacientes portadores do vírus HIV, sem tratamento, podendo variar de 20 a $83 \%$, assim como portadores da doença de Parkinson, também não tratados. ${ }^{(2)}$

A etiologia da DS não está totalmente elucidada, mas há um consenso que três fatores são importantes no desenvolvimento da doença: secreção das glândulas sebáceas, alterações da microbiota (Malassezia $s p$ ) da pele e resposta imune do indivíduo. ${ }^{(3)}$

Pacientes com DS não apresentam necessariamente glândulas sebáceas hipersecretoras, mas podem apresentar diferenças quantitativas na composição 
NETO, E. M. R.; MARQUES, L. A. R. V.; LOTIF, M. A. L.; COELHO, M. O.; NOCRATO, M. N.; RODRIGUES, J. C.

Revista Eletrônica de Farmácia Vol. X (4), 16 - 26, 2013.

do sebo presente na pele, o que levaria a um favorecimento da colonização por micro-organismos lipídios-dependentes. (4)

O papel das leveduras do gênero Malassezia ainda é controverso, pois são espécies comensais geralmente isoladas de áreas do corpo ricas em sebo como face, couro cabeludo, tronco e peitoral. É aceito que a produção de lipases, pelo comensal, causa a hidrólise de triglicerídeos e ácidos graxos livres gera metabólitos irritantes e pró-inflamatórios de forma abundante, mantendo a natureza característica da doença. ${ }^{(5)}$

Em relação à imunidade, os pacientes com DS não demonstram níveis de anticorpos maiores que indivíduos não portadores, mas apresentam maiores níveis de células NK (Natural Killers), CD $16^{+}$, interleucinas inflamatórias e atividade do sistema complemento no tecido lesionado que no tecido sadio. $(3,6)$

Tendo em vista que a DS é uma condição patológica sem cura definitiva cabe ao farmacêutico esclarecer ao paciente que os objetivos do tratamento são controlar os sinais visíveis das lesões (descamação e eritema) e sintomas incômodos como o prurido. ${ }^{(3)} \mathrm{O}$ paciente também deve ser informado da condição crônica e recidivante da doença e das situações desencadeadoras de crises (estresse, uso de formulações queratolíticas fortes e irritantes) para dessa forma favorecer o manejo das lesões. $(7,8)$

Em agosto de 2013 foi publicada pelo Conselho Federal de Farmácia a resolução No 586, que entre outras providências regula a prescrição farmacêutica, elevando o farmacêutico ao posto de profissional responsável pelo gerenciamento farmacoterapêutico de males menores de saúde e de outras patologias de interesse de saúde pública em caráter complementar. ${ }^{(9)}$

É importante resaltar que em situações atípicas o farmacêutico deve encaminhar, de forma documentada, o paciente a um atendimento médico: quando pertencer a um grupo de risco para uso de medicamentos (gestantes, nutrizes, crianças, recém-nascidos e idosos); quando o problema de saúde não puder ser tratado com o arsenal terapêutico disponível, legalmente, para a prescrição farmacêutica e quando os sintomas apresentados forem provenientes de outra patologia mais complexa.

O estudo poderá contribuir para com os profissionais farmacêuticos nas ações de gerenciamento farmacoterapêutico, bem como para com os portadores de DS no aprimoramento da abordagem terapêutica, através do levantamento do que se tem pesquisado em estudos científicos.

\section{OBjetIVO}


NETO, E. M. R.; MARQUES, L. A. R. V.; LOTIF, M. A. L.; COELHO, M. O.; NOCRATO, M. N.; RODRIGUES, J. C.

Revista Eletrônica de Farmácia Vol. X (4), 16 - 26, 2013.

Realizar uma revisão de literatura visando nortear a abordagem terapêutica da DS no âmbito competente da clínica farmacêutica.

\section{MÉTODOS}

Foi realizada uma revisão de literatura com pesquisa nos bancos de dados do Pubmed, Bireme, Lilacs e Scielo, bem como pesquisa nos periódicos fornecidos pelo Portal Brasileiro da Informação Científica sobre o assunto. Foram usados os descritores:

Dermatite Seborréica, Tratamento Farmacológico e Malassezia. Essa pesquisa foi realizada durante o mês de Outubro de 2013, dos estudos encontrados foram selecionados 26 artigos publicados entre os anos de 1991 e 2012; estes foram relacionados à abordagem terapêutica da DS tendo como foco a competência profissional do farmacêutico, no âmbito da sua clínica.

\section{REVISÃO DA LITERATURA}

Tendo a em vista que a DS se trata de uma doença com natureza inflamatória e caráter crônico, sendo esta correlacionada a presença de leveduras do gênero Malassezia e seu metabolismo lipídio-dependente, o tratamento terá 3 vias de ação viáveis: o controle da inflamação, da proliferação do patógeno e da oleosidade epidérmica. (10)

CONTROLE DA INFLAMAÇÃO
Os corticosteróides tópicos são uma alternativa terapêutica bastante viável, pois levam a uma melhora rápida dos sintomas: eritema, descamação e prurido. Porém, quando são interrompidos geram frequentes recidivas; devendo, também, ser utilizados durante $o$ menor tempo possível em virtudes dos efeitos colaterais que ocorrem com o uso prolongado. ${ }^{(11)}$

O controle da inflamação de caráter crônico pode ser alcançado pelo uso de corticosteróides de baixa potência em formulações tópicas (12), sendo a Hidrocortisona o único corticoide de dispensação isenta de prescrição. (13)

Em um estudo duplo-cego com 72 voluntários, comparando a eficácia da Hidrocortisona creme $1 \% \mathrm{p} / \mathrm{p}$ com Cetoconazol creme $2 \% \mathrm{p} / \mathrm{p}$, com DS leve a moderada foi encontrado que ambos os medicamentos apresentaram resultados semelhantes na diminuição dos sintomas de descamação, rubor, coceira e pápulas. ${ }^{(14)}$

Num outro estudo duplo-cego, randomizado e comparativo com duração de 12 semanas; no qual foi testada Hidrocortisona unguento $1 \% \mathrm{p} / \mathrm{p}$ frente ao tacrolimus pomada $0,1 \%$ (Inibidor da calcineurina, que age por uma via antiinflamatória distinta, gerando, também uma imunomodulação) também não foram encontradas diferenças 
NETO, E. M. R.; MARQUES, L. A. R. V.; LOTIF, M. A. L.; COELHO, M. O.; NOCRATO, M. N.; RODRIGUES, J. C.

Revista Eletrônica de Farmácia Vol. X (4), 16 - 26, 2013.

significantes durante a avaliação médica. (15)

Há um consenso que os
corticosteróides tópicos são úteis nos
tratamentos de curto prazo, principalmente para controlar o eritema e o prurido, assim como a descamação. Não ha dados disponíveis para resolver a questão da maior efetividade da combinação de corticosteróides e agentes antifúngicos tópicos frente a monoterapia com um agente isolado. $\mathrm{O}$ uso por períodos prolongados gera preocupação devido seus efeitos adversos locais como a atrofia da pele e hipertricose. ${ }^{(16)}$

CONTROLE DA PROLIFERAÇÃO DO PATÓGENO

É notório o importante papel do metabolismo de leveduras do gênero Malassezia na DS, assim, a utilização de agentes antifúngicos recebe destaque na abordagem terapêutica dessa condição. (17)

Ao realizar a seleção da farmacoterapia com agentes antifúngicos o farmacêutico terá um amplo arsenal terapêutico, sendo viável a prescrição de uma variada gama de tratamentos tópicos. $(9,13)$

O enxofre, por apresentar propriedades antifúngicas e queratolítica é uma opção clínica plausível, podendo ser associado ao ácido salićlico para atuar de maneira sinérgica, quando veiculado em formulações como sabonetes e xampus, tendo apenas a desvantagem do seu odor residual característico. ${ }^{(18)}$ O Sabonete com óleo de Melaleuca alternifólia (tea tree oil) a $5 \% \mathrm{p} / \mathrm{p}$ foi uma formulação que se mostrou, também, bastante promissora por apresentar bom potencial de atividade antimicótica. ${ }^{(19)}$

Outros agentes fungistáticos são empregados com sucesso em fórmulas de xampus como piritionato de zinco e o sulfeto de selênio em concentrações que variam de 1 a 2,5\%, ambos apresentaram efeitos queratolítico que podem ter sua ação potenciada com a associação do ácido salicílico na formulação, facilitando, desse modo, a remoção das escamas e propiciando um efeito anti-inflamatório sobre as lesões. (18)

Os agentes antifúngicos azólicos se apresentam como outra opção terapêutica bastante útil, dentre eles destaca-se o cetoconazol, que apresenta também alguma atividade antiinflamatória, possivelmente, por inibição da 5-Lipooxigenase, tendo repercussão na síntese de leucotrienos e outros derivados do ácido araquidônico. Outra atividade de interesse clínico do cetoconazol e sua, provável, capacidade de regular a secreção lipídica pelas glândulas sebáceas. Haja vista que a secreção sebácea alterada é uma característica marcante da DS. Por fim 
NETO, E. M. R.; MARQUES, L. A. R. V.; LOTIF, M. A. L.; COELHO, M. O.; NOCRATO, M. N.; RODRIGUES, J. C.

Revista Eletrônica de Farmácia Vol. X (4), 16 - 26, 2013.

esse fármaco apresenta algum efeito antiproliferativo, atuando na diferenciação dos queratinócitos humanos, sendo essa uma vantagem no controle da descamação. ${ }^{(20)}$

Dados de um ensaio clínico demonstraram que quando aplicado, em vários locais do corpo, duas vezes por dia durante quatro semanas, 0 cetoconazol $2 \% \mathrm{p} / \mathrm{p}$ creme, demonstrou ser tão eficaz quanto à hidrocortisona $1 \% \mathrm{p} / \mathrm{p}$ creme no tratamento da DS. ${ }^{(20)}$

Em outro estudo randomizado, duplo-cego com 459 pacientes com DS tratados com cetoconazol $2 \%$ gel ou veículo uma vez ao dia durante 14 dias, houve uma taxa significativamente maior de sucesso do tratamento, bem como, maior redução no eritema, prurido e descamação nos pacientes tratados com cetoconazol. (21)

Nos casos de DS no couro cabeludo, a caspa, foi demonstrado que o ciclopirox olamina xampu é uma alternativa interessante, tendo em vista dados de um ensaio clínico, randomizado, duplo-cego, controlado, que participaram 949 pacientes, que utilizavam o produto 1 ou 2 vezes por semana durante 4 semanas e apresentaram melhores resultados que o grupo controle. Também foi verificado que o uso do xampu 1 vez por semana ou a cada 2 semanas diminuiu a incidência de crises nos pacientes. ${ }^{(22)}$ É interessante resaltar, também, que estudos relatam um efeito antiinflamatório do ciclopriox olamina comparável à hidrocortisona, ao atuar de modo semelhante ao cetoconazol inibindo ação a 5-Lipooxigenase e Ciclooxigenases. (23)

A eficácia do cilopirox olamina xampu foi comparada com cetoconazol xampu em um estudo duplo-cego com 350 pacientes com DS. Os dois tratamentos foram igualmente eficazes e melhores que o placebo, entretanto os pacientes classificaram 0 ciclopirox olamina xampu como sendo um tratamento mais favorável. (24)

\section{CONTROLE DA OLEOSIDADE EPIDÉRMICA}

Como citado anteriormente fungos do gênero Malassezia desempenham seu papel na patogênese da DS por um mecanismo lipídio dependente, tendo em vista esse dado é possível afirmar que o controle da oleosidade será uma via de tratamento possível para o controle da doença, principalmente no que tange o seu caráter inflamatório. ${ }^{(25)}$

Formulações contendo coaltar ou seus derivados apresentam múltiplos benefícios ao paciente portador de DS, pois sua ação se dará por 3 mecanismos distintos: controle da oleosidade, xampus e sabonetes apresentarão alto poder adstringente, deixando, desse modo a epiderme com uma quantidade significativamente menor de sebo; efeito 
NETO, E. M. R.; MARQUES, L. A. R. V.; LOTIF, M. A. L.; COELHO, M. O.; NOCRATO, M. N.; RODRIGUES, J. C.

Revista Eletrônica de Farmácia Vol. X (4), 16 - 26, 2013.

citostáticos, atuando na diferenciação celular e consequentemente na formação de escamas; e uma ação antimicótica, ao controlar a proliferação da população fúngica residente. Em ensaio clínico randomizado o xampu de coaltar a $4 \%$ $\mathrm{p} / \mathrm{p}$, quando comparado com o placebo, mostrou alta taxa de redução da caspa nos pacientes. $(18,26)$

A prescrição farmacêutica de fitoterápicos tópicos veiculados em xampu ou sabonetes é uma opção clínica razoável, tendo em vista o melhor perfil de efeitos adversos e eficácia comprovada. Podem-se referir formulações contendo extrato de Hamamelis virginiana e Pilocarpus jaborandi, ambos em concentração de
$3 \%$, podendo ser combinados. Elas apresentaram propriedades adstringentes, anti-inflamatórias, antioxidantes, antissépticas e controladoras de oleosidade. (27)

Vale ressaltar que o farmacêutico também tem o papel de informar ao paciente dos cuidados inadequados $\mathrm{e}$ fatores desencadeadores de crise de DS, como por exemplo: uso excessivo de condicionadores próximo ao couro cabeludo e enxágue inadequado, uso de água muito quente (que causa ressecamento excessivo e gera um efeito rebote de mais produção de sebo), excesso de exposição ao sol, situações de fadiga ou estresse emocional e ingestão de alimentos gordurosos, bebidas alcoólicas e fumo. ${ }^{(28)}$

Tabela 01: Consolidado de possíveis opções terapêuticas, na clínica farmacêutica, para tratamento de Dermatite Seborréica e suas respectivas vias de ação.

\begin{tabular}{llcl}
\hline & \multicolumn{3}{c}{ Via de Ação } \\
\hline \multirow{2}{*}{ Opção Terapêutica } & Controle da & Controle da & Controle da \\
& Inflamação & Proliferação Fúngica & Oleosidade \\
\hline
\end{tabular}

Hidrocortisona

creme $1 \%$

$X$

Formulações com

Enxofre, Selênio e

$x$

X

Zinco

Formulações com

Ácido Salicílico

X

$x$

Formulações com

Cetoconazol

$x$

$X$

X

Xampu de ciclopirox

olamina

X

X

Formulações com

$x$

X

X 
NETO, E. M. R.; MARQUES, L. A. R. V.; LOTIF, M. A. L.; COELHO, M. O.; NOCRATO, M. N.; RODRIGUES, J. C.

Revista Eletrônica de Farmácia Vol. X (4), 16 - 26, 2013.

Coaltar ou derivados

Formulações com

Hamamelis

virginiana ou

$X$

$\mathrm{X}$

Pilocarpus jaborandi

Formulações com

Melaleuca

alternifólia (tea tree

$x-x$

oil)

\section{CONSIDERAÇÕES FINAIS}

A Dermatite Seborréica é doença muito comum e recorrente, caracterizada por cronicidade, inflamação e descamação epidérmica e distribuída em ampla faixa etária. A abordagem terapêutica deve seguir 3 vias de ação: controle da inflamação, controle da proliferação fúngica e controle da oleosidade epidérmica.

Tendo em vista que, muitas vezes, o farmacêutico será o primeiro e, provavelmente, o mais acessível profissional de saúde que o paciente procura é de suma importância que, dentro da sua competência clínica, ele seja capaz de resolver um problema de saúde menor ou encaminhar o paciente para atendimento específico, quando necessário. Nesse contexto é imprescindível que o profissional conheça dentro de seu âmbito de atuação suas opções de farmacoterapia, bem como a efetividade do arsenal disponível.

É valido ressaltar a extrema importância do acompanhamento por utilização de métodos de atenção farmacêutica do paciente com medidas não farmacológicas como indicação de rotinas de higiene adequadas e mudanças em hábitos de vidas não condizentes com sua condição clínica.

Desse modo, somado como os esforços próprios do paciente, são possíveis de obter-se um controle efetivo e um maior espaçamento na recorrência das crises da doença.

\section{REFERÊNCIAS}

1. Ferolla, C. Dermatite seborréica da face. Rev Bras Med Dermatol 2010; 67: 11-5 
NETO, E. M. R.; MARQUES, L. A. R. V.; LOTIF, M. A. L.; COELHO, M. O.; NOCRATO, M. N.; RODRIGUES, J. C.

Revista Eletrônica de Farmácia Vol. X (4), 16 - 26, 2013.

2. Schechtman RC, Midgley G, Hay RJ. HIV disease and Malassezia yeasts: a quantitative study of patients presenting with seborrhoeic dermatitis. $\mathrm{Br} J$ Dermatol.1995;133:694-8.

3. Del Rosso JQ. Adult seborrheic dermatitis: a status report on practical topical management. J Clin Aesthet Dermatol. 2011;4(5):32-8.

4. De Luca C, Valacchi G. Surface lipids as multifunctional mediators of skin responses to environmental stimuli. Mediators Inflamm. 2010;2010:321494.

5. Dawson TL Jr. Malassezia globosa and restrica: breakthrough understanding of the etiology and treatment of dandruff and seborrheic dermatitis through whole-genome analysis. J Invest Dermatol Symp Proc. 2007;12:15-19

6. Sampaio AL, Mameri AC, Vargas TJ, et al. Seborrheic dermatitis. An Bras Dermatol. 2011;86:1061-74.

7. Peyri J, Lleonart M, and the Spanish Group of the SEBDERM Study. Clinical and therapeutic profile and quality of life of patients with seborrheic dermatitis. Actas Dermosifiliogr. 2007;98:476-82.

8. Stefanaki I, Katsambasa A. Therapeutic update on seborrheic dermatitis. Skin Therapy Lett. 2010;15(5):1-4.

9. Conselho Federal de Farmácia. Resolução no 586, de 29 de agosto de 2013. Regula a prescrição farmacêutica e dá outras providências

10. Elewski B. Safe and effective treatment of seborrheic dermatitis. Cutis. 2009;83:333-8

11. Sampaio ALB, Mameri A, Jeunon T, Ramos-e-Silva M, Nunes AP, Carneiro S. Dermatite seborreica. An Bras Dermatol. 2011;86(6):1061-74

12. Goldenberg, G. Optimizing Treatment Approaches in Seborrheic Dermatitis. The Journal of Clinical and Aesthetic Dermatology. 2013;6(2): 44-9

13. BRASIL. Agência Nacional de Vigilância Sanitária. Resolução RDC no 138, de 29 de maio de 2003. Dispõe sobre o enquadramento na categoria de venda de Medicamentos.

14. Stratigos JD, Antoniou C, Katsambas A, et al. Ketoconazole $2 \%$ cream versus hydrocortisone $1 \%$ cream in the treatment of seborrheic dermatitis. A double-blind comparative study. J Am Acad Dermatol. 1988;19:850-3.

15. Papp KA, Papp A, Dahmer B, Clark CS. Single-blind, randomized controlled trial evaluating the treatment of facial seborrheic dermatitis with hydrocortisone $1 \%$ ointment compared with tacrolimus $0.1 \%$ ointment in adults. J Am Acad Dermatol. 2012;67:11-5.

16. Naldi L e Rebora A. Seborrheic Dermatitis. N Engl J Med 2009;360:387-96 
NETO, E. M. R.; MARQUES, L. A. R. V.; LOTIF, M. A. L.; COELHO, M. O.; NOCRATO, M. N.; RODRIGUES, J. C.

Revista Eletrônica de Farmácia Vol. X (4), 16 - 26, 2013.

17. Elewski BE, Abramovits W, Kempers $S$, et al. A novel foam formulation of ketoconazole $2 \%$ for the treatment of seborrheic dermatitis on multiple body regions. J Drugs Dermatol. 2007;6(10):1001-08.

18. Wolwerton SE, editor. Compreensive dermatologic drug therapy. 2nd ed. Philadelphia: Saunders Elsevier; 2007. p.720-3.

19. Satchell AC, Saurajen A, Bell C, Barnetson RS. Treatment of dandruff with $5 \%$ tea tree oil shampoo. J Am Acad Dermatol. 2002;47:852-5

20. Borgers, M.; Degreef, H. The Role of Ketoconazole in Seborrheic Dermatitis. Cutis. 2007 80(4):359-63.

21. Elewski B, Ling MR, Phillips TJ. Efficacy and safety of a new once-daily topical ketoconazole $2 \% \mathrm{gel}$ in the treatment of seborrheic dermatitis: a phase III trial. J Drugs Dermatol. 2006;5(7):646-50.

22. Shuster S, Meynadier J, Kerl H, Nolting S. Treatment and prophylaxis of seborrheic dermatitis of the scalp with antipityrosporal 1\% ciclopirox shampoo. Arch Dermatol 2005; 141:47-52.

23. Hanel H, Smith-Kurtz E, Pastowsky S. [Therapy of seborrheic eczema with an anti fungal agent with an antiphlogistic effect]. Mycoses. 1991;34(1):91-3.

24. Ratnavel RC, Squire RA, Boorman GC. Clinical efficacies of shampoos containing ciclopirox olamine $(1.5 \%)$ and ketoconazole $(2.0 \%)$ in the treatment of seborrhoeic dermatitis. J Dermatol Treat. 2007;18(1):88-96.

25. Picardo M, Cameli N. Seborrheic dermatitis. In: Williams H, Bigby M, Diepgen T, Herxheimer A, Naldi L, Rzany B, eds. Evidence-Based Dermatology, Second Edition. Malden, Massachusetts: Blackwell Publishing; 2008:164-70.

26. Davies DB, Boorman GC, Shuttleworth D. Comparative efficacy of shampoos containing coal tar $(4.0 \% \mathrm{w} / \mathrm{w}$; Tarmed), coal tar $(4.0 \% \mathrm{w} / \mathrm{w})$ plus ciclopirox olamine $(1.0 \% \mathrm{w} / \mathrm{w}$; Tarmed) and ketoconazole $(2.0 \% \mathrm{w} / \mathrm{w}$; Nizoral) for the treatment of dandruff/seborrhoeic dermatitis. J Dermatol Treat 1999;10(3):177-83.

27. Souza, VM, Antunes Junior, D. Ativos Dermatológicos. São Paulo: Pharmabooks, 2009.

28. Formariz, TP, Spera, LJ, Urban, MCC, Gremiao, M.P.D. Dermatite seborréica: causas, diagnóstico e tratamento. Infarma. 2005; 16(13/14):77-80. 